\title{
Germline variant
}

National Cancer Institute ( $\mathrm{NCl})$

\section{Source}

National Cancer Institute (NCI). Germline variant.

A gene change in a reproductive cell (egg or sperm) that becomes incorporated into the DNA of every cell in the body of the offspring. A variant contained within the germline can be passed from parent to offspring, and is, therefore, hereditary. 